\title{
Scoping a Vocabulary for Spatial Relations Properties
}

\author{
Dalia E. Varanka * \\ U.S. Geological Survey, dvaranka@usgs.gov \\ * Corresponding author
}

Keywords: Spatial Relations, Geospatial Ontology, Controlled Vocabulary

\begin{abstract}
:
Spatial relations are essential for knowledge representation, yet the scope of a corpus of geospatial terms, such as exists for RDF or OWL, is not yet recognized. A vocabulary of geospatial relations may align with several existing models within RDF and OWL; among which are relation primitives as defined in upper ontology; regional topological relations such as those expressed by the Open Geospatial Consortium (OGC) GeoSPARQL standard; and mereotopologic relations as are researched in related semantic literature. One semantic area that is theorized among linguists, but not well defined within formal logic are verb-preposition combinations. The objective of this study in-progress is to 1 . Define a corpus of spatial relation terms, 2. Place such relation classes within a framework of existing semantic axioms, and 3. To identify the types of spatial relation terms that need more research. To understand and enrich the vocabulary of geospatial feature properties for semantic technology, English language spatial relation predicates were analysed in three standard topographic feature glossaries. Five major classes of spatial relation predicates were identified from the analysis. First, part-whole relations are modelled throughout semantic and linked-data networks. The remaining classes are spatially descriptive and geometric relations; physical processes happening in space; human use of geographic space, such as land use; and spatial preposition spatial relations. These categories are commonly found in the 'real world' and support environmental science based on digital topographical mapping. The hypothesis is that a broad set of spatial relation expressions, form the basis for expanding the range of possible queries for topographical data and mapping applications.
\end{abstract}

